EPJ Web of Conferences 75, 05016 (2014)

DOI: 10.1051/epjconf/20147505016

(C) Owned by the authors, published by EDP Sciences, 2014

\title{
Temperature dependence of ferromagnetic resonance measurements in nanostructured line arrays
}

\author{
V. Raposo. ${ }^{1}$, A.G. Flores ${ }^{1}$, M. Zazo ${ }^{1}$, J. Iñiguez. ${ }^{1}$, C. Redondo ${ }^{2}$, D. Navas ${ }^{2}$ \\ ${ }^{1}$ Departamento de Física Aplicada, Universidad de Salamanca, Plaza de la Merced s/n, 37008 Salamanca, España \\ ${ }^{2}$ Departamento de Química Física, Universidad del País Vasco, Barrio Sarriena s/n, 48940 Lejona (Vizcaya), España
}

\begin{abstract}
We report the effect of temperature on the ferromagnetic resonance (FMR) spectra of nanostructured line arrays. Different temperature dependences are observed for permalloy an nickel based samples. The qualitative features of the temperature dependence of the resonance field and linewidth can be described by the usual expression of slow relaxing linewidth mechanism and Bloch equation.
\end{abstract}

\section{Introduction}

Dynamic properties of magnetic nanostructures have recently been intensively studied due to their potential application in high density perpendicular recording media, magnetic sensors or microwave devices [1-4]. To understand magnetization processes in these materials it should be considered the intergrain exchange coupling, magnetic dipolar interactions and the anisotropy fields. The anisotropies fields can be investigated by various techniques. One of these techniques is the ferromagnetic resonance spectroscopy (FMR) due to the fact that the resonance field depends directly on the anisotropy field strength and its angular spread [5], [6].

\section{Experimental}

Nanostripe arrays of $\mathrm{Ni}$ and Permalloy have been prepared by interference lithography (IL). Interference lithography technique has been shown as an effective tool for the fabrication of templates with periodic nanostructures, such as line arrays, in large areas $\left(\mathrm{cm}^{2}\right)$ [7]. Our IL system uses a Lloyd's mirror configuration and a He-Cd TEM00 laser (KimmonElectric Co.) with a fixed wavelength of $325 \mathrm{~nm}$. For the fabrication of the templates, a stack of $\approx 80 \mathrm{~nm}$ thick anti-reflection-coating (WiDE-8B, Brewer Science, Inc.) and $\approx 200 \mathrm{~nm}$ thick negative-tone-resist (TSMR-iN027, Brewer Science, Inc.) was spun sequentially on (100) $\mathrm{Si}$ wafers with native oxide. After exposure, the templates were baked at $110^{\circ} \mathrm{C}$ during $90 \mathrm{~s}$ and developed in AZ726 MIF (Microchemicals). A polycrystalline film is deposited on the template by using Ion Beam Sputtering system (IBS) [8]. Finally, the line array was achieved after a liftoff process by immersing the samples in 1-methyl2pyrrolidinone (NMP) at $120^{\circ} \mathrm{C}$.
The film thickness of nanostripe arrays of $\mathrm{Ni}$ is of $45 \mathrm{~nm}$ for a lattice period of $2.7 \mu \mathrm{m}$ and the stripe width of 1500 $\mathrm{nm}$ for sample NiL1 and of $750 \mathrm{~nm}$ for sample NiL2. The film thickness of Permalloy arrays is of $20 \mathrm{~nm}$ for a lattice period of $2.9 \mu \mathrm{m}$ and the stripe width of $1331 \mathrm{~nm}$ for sample PyL1 and of $1397 \mathrm{~nm}$ for sample PyL2.

Ferromagnetic resonance (FMR) measurements have been performed as a function of temperature at a frequency of $9.75 \mathrm{GHz}$ with a narrowband FMR spectrometer using an Agilent E5071C network analyzer as a source and tuning unit connected to a Bruker rectangular resonant cavity model ERE4112ST and a diode as receiver of the reflected field-modulated microwave signal [9] measured by a SR830 lock-in amplifier. The amplitude of the modulation field and its frequency have been $32 \mathrm{Oe}$ and $20 \mathrm{kHz}$ respectively. Samples were placed in a Oxford Instruments continuous flow cryostat and FMR was measured from 85 to $300 \mathrm{~K}$. Temperature stability was better than $0.2 \mathrm{~K}$.

\section{Results}

Figure 1 shows the FMR spectra as a function of the magnetic field for several temperatures for $\mathrm{Ni}$ based samples. Resonance field is obtained from the maximum second derivative of the absorbed power respect to the magnetic field and linewidth is the magnetic field difference between maximum and minimum at $\mathrm{dP} / \mathrm{dH}$. From the study of these curves we can obtain the temperature dependence of both resonance field and linewidth. As observed in figure 2 linewidth is almost constant in temperature, with only a small decrease, while resonance field presents a uniform decay as temperature increases. 
In order to study these results we use the relationship from resonance frequency and magnetic field:

$$
(\omega / \gamma)^{2}=\left[H+\left(2 K_{1} / M_{s}\right)\right] \times\left[H+4 \pi M_{s}+\left(2 K_{1} / M_{s}\right)\right]
$$

with $M_{S}$ the saturation magnetization, $K_{I}$ the anisotropy constant an $\gamma$ the gyromagnetic ratio. Anisotropy field $\left(H_{k}\right)$ is defined as $2 K_{l} / M_{S}$. This equation can be simplified because $M_{S}$ is usually much higher than the applied and anisotropy field. In that case:

$$
(\omega / \gamma)^{2} \approx\left[H+\left(2 K_{1} / M_{s}\right)\right] M_{s}
$$
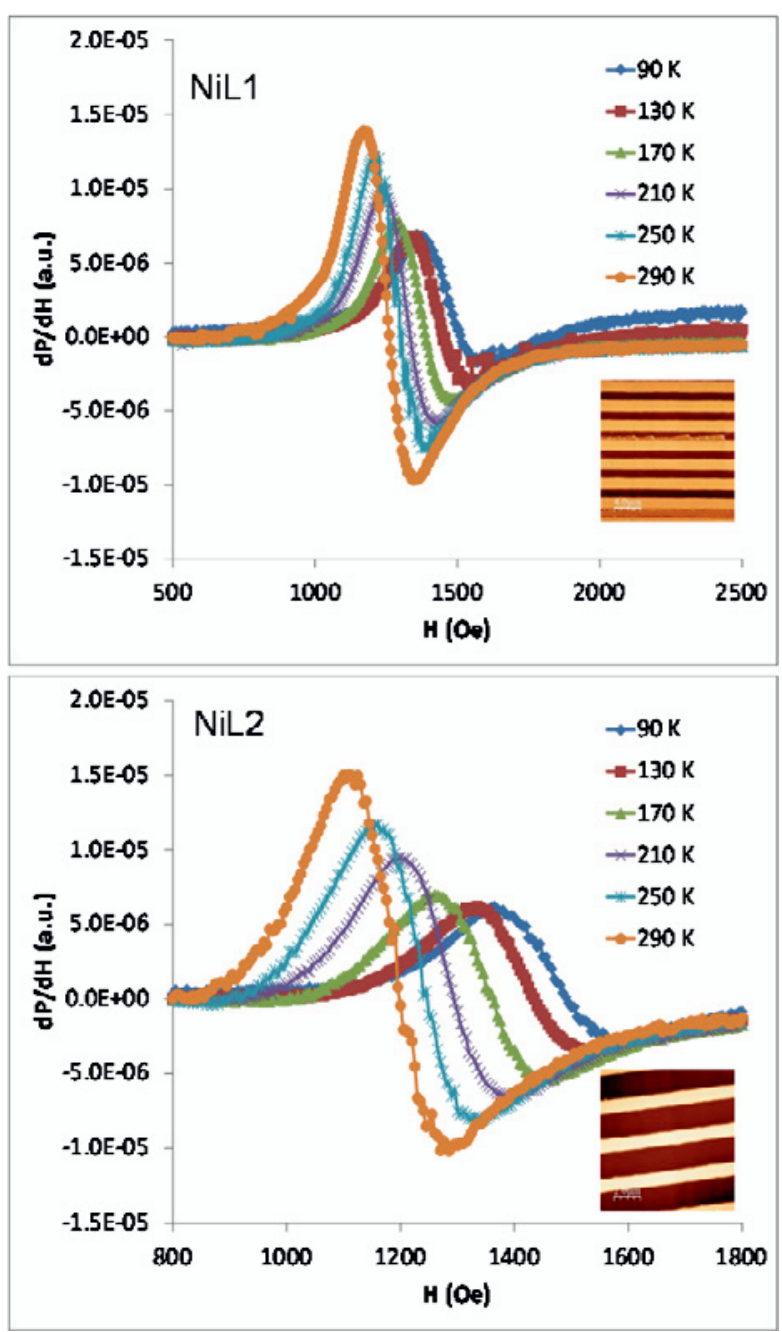

Fig. 1. FMR spectra for samples NiL1 and NiL2 at several temperatures.

Equation (1) is valid for planar samples with in-plane anisotropy and magnetic field along the easy axis [10]. Taking into account also Bloch equation:

$\frac{M_{S}(T)}{M_{S}(0)}=1-\beta T^{3 / 2}$

the temperature dependence of resonance field can be explained in the following way: as resonance field for a fixed frequency depends on saturation magnetization and anisotropy constant for a fixed frequency, taking into account the temperature dependence of the saturation magnetization of $\mathrm{Ni}$ based samples from ecuation (2), resonance field should increase about $120 \mathrm{Oe}$, and the decrease of the experimental value reveals a decrease in the anisotropy constant with temperature. While linewith is very similar for both samples only a slight difference on resonance field can be found. As magnetic field is applied along the lines where demagnetization factor is negligible, differences on resonance field are caused by by misalignment of the sample plane to the magnetic field

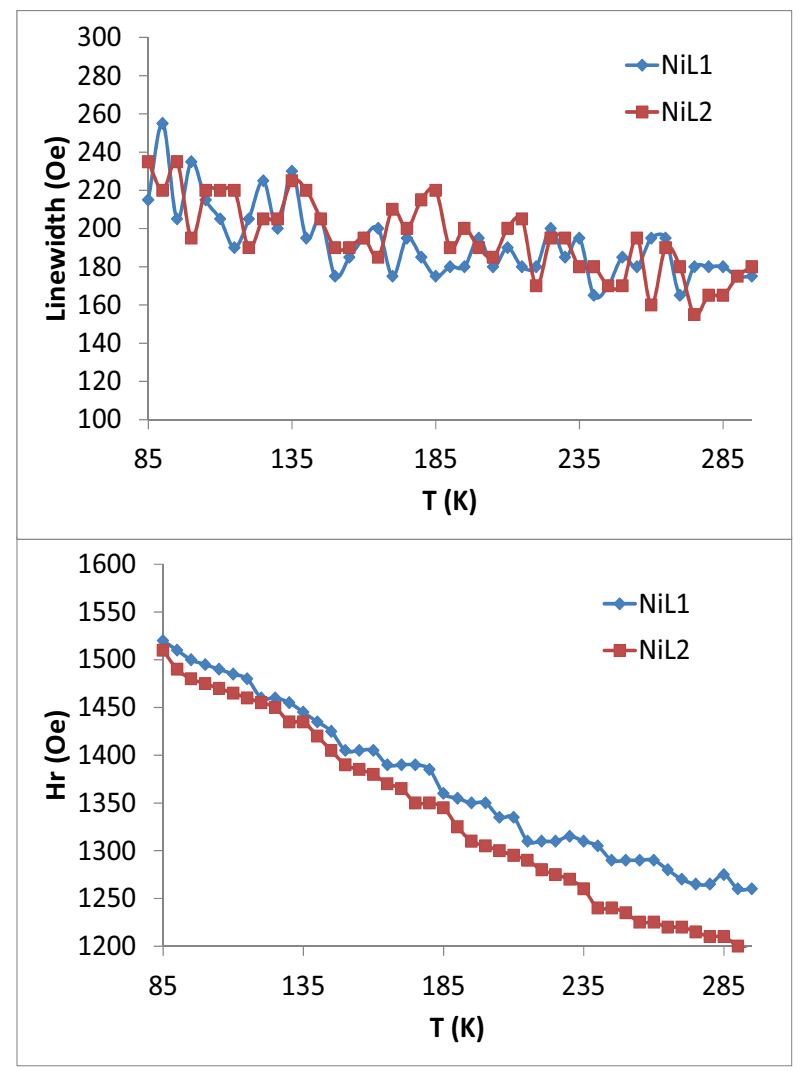

Fig. 2. Temperature dependence of linewith and resonance field for samples NiL1 and NiL2.

Similar analysis is performed in permalloy based samples. We plot FMR spectra for samples PyL1 and PyL2 in figure 3, were we can see that samples present an opposite temperature dependence of the resonance field as compared to nickel based samples. This is clearly showed in figure 4 where we can see a perfectly constant linewidth. The value is slightly higher than the linewith in bulk samples but in accordance to the increased linewith described in thin films [11]. For permalloy based samples resonance field increases with temperature. In this samples and using Bloch equation (3) the temperature dependence of saturation magnetization explains the increase of resonance field with a constant anisotropy constant by means of equation (1). Differences between PyL1 and PyL2 are again mainly caused by misalignment of the sample plane to the magnetic field, as geometric characteristics are almost identical. 

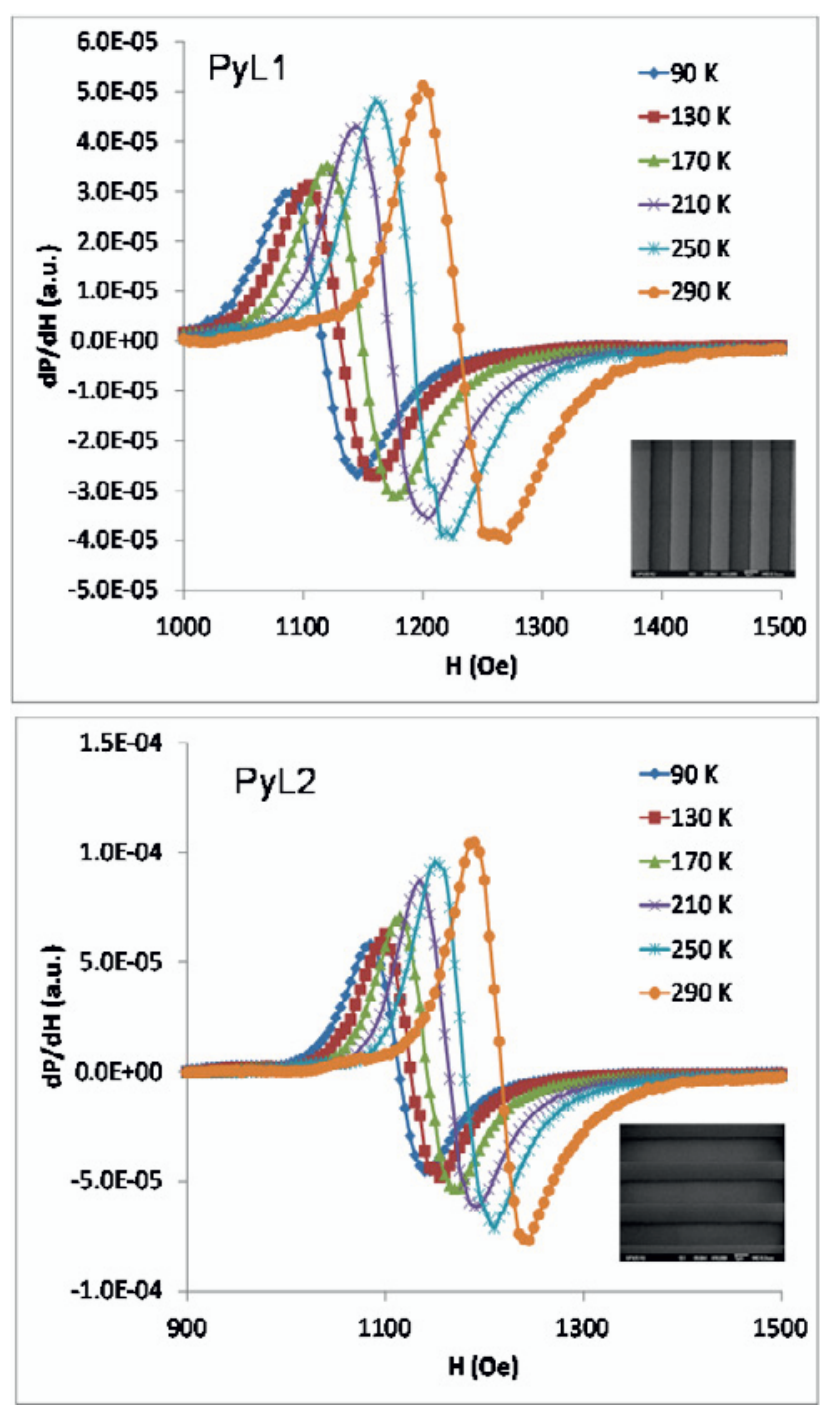

Fig. 2. FMR spectra for samples PyL1 and PyL2 at several temperatures.

\section{Conclusions}

Both Nickel and Permalloy based samples present a nearly constant linewidth. This suggest that in the 85-300 $\mathrm{K}$ range the losses in the samples are almost kept constant, with a small temperature dependence of the resistivity.

Resonance field presents a opposite temperature dependence in each composition, decreasing with temperature in Nickel based samples, and increasing for the Py. As resonance field for a fixed frequency depends on saturation magnetization and anisotropy constant, taking into account the temperature dependence of the saturation magnetization of $\mathrm{Ni}$ based samples, resonance field should increase about $120 \mathrm{Oe}$, and the decrease of the experimental value reveals a decrease in the anisotropy constant with temperature. For Py samples the increase of the resonance field is explained by the decrease of saturation magnetization with temperature due to the small value of anisotropy constant

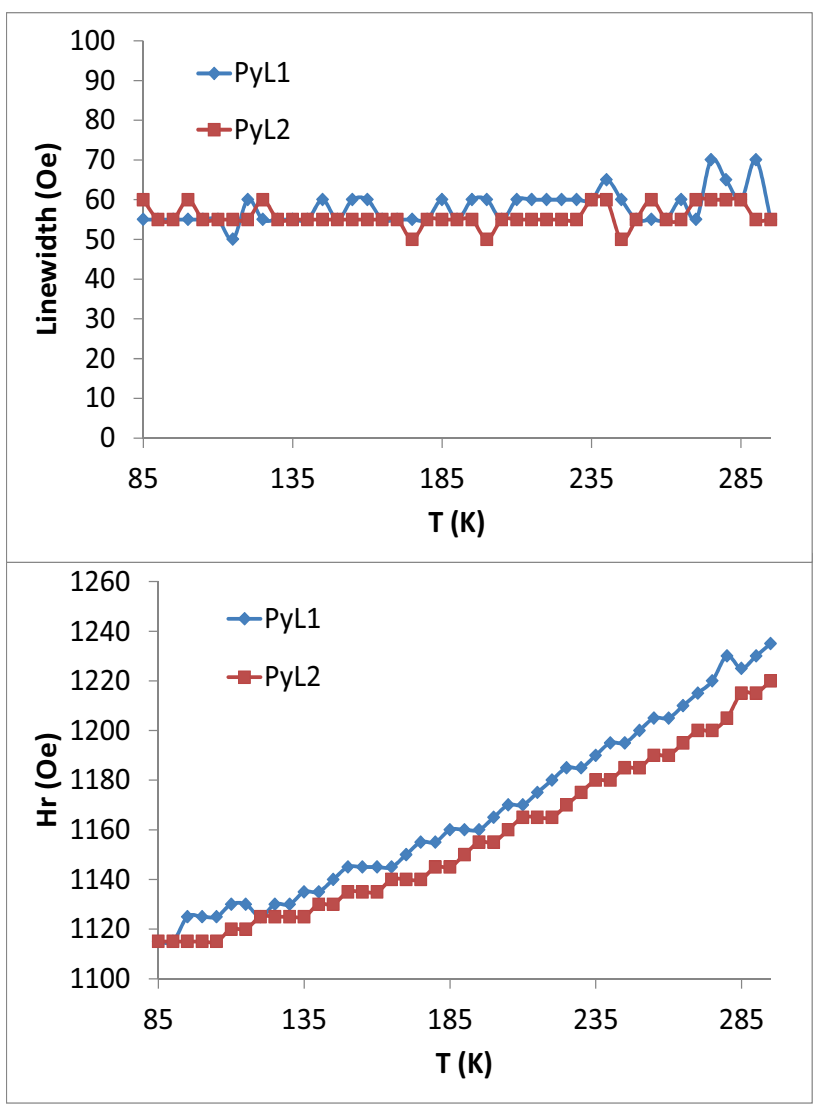

Fig. 4. Temperature dependence of linewith and resonance field for samples PyL1 and PyL2.

\section{Acknowledgments}

This work was supported in part by the Ministerio de Educación y Ciencia of Spain under project MAT201020798-C05-05 with FEDER support.

\section{References}

1. K. J. Merazzo, R. P. del Real, A. Asenjo, and M. Vazquez, J. App. Phys. 109, 07B906 (2011)

2. C. T. Boone et al, Phys. Rev. Lett., 103, 167601, (2009)

3. Dumitru et al, IEEE Trans. Magn., 41, 3361 (2005)

4. D. Hinzke and U. Nowak, J. Magn. Mag. Mat., vol 221, 365 (2000)

5. A Popa, M Jivanescu, D Toloman, O Raita and L M Giurgiu, J. Phys.: Conf. Ser., 182, 012073 (2009)

6. Ghaddar et al., Act. Phys. Pol. A, 116, 1039 (2009)

7. M. E. Walsh, "Ph. D. Thesis", Massachusetts Institute of Technology (2000)

8. C. Redondo et al, Nanotechnology, 17, 2040 (2006)

9. A. G. Flores, V. Raposo, J. Iñiguez, M. Zazo, C. Redondo, and D. Navas, IEEE Trans. Magn, 49, 15 (2013)

10. O. Kohmoto, J. Magn. Mag. Mat., 262, 280 (2003)

11. P. Lubitz, M. Rubinstein, J.J. Krebs and S.-F. Cheng, J.App. Phys, 89, 6901 (2001) 